\title{
NOTES DE VOYAGE AUX ÉTATS-UNIS
}

(Suite.)

\section{LAMINAGE DES BILLETTES, LARGETS ET FERS MARCHANDS}

PAR

\section{F. CORVEE}

Les différents systèmes de fabrication de la billette aux États-Unis, au moins dans les installations récentes, difrèrent beaucoup de la méthode européenne, et présentent quelques particularités intéressantes à signaler.

La fabrication des gros blooms proprement dits et vendus comme tels à des industries de dénaturation, tend à disparaître; la majeure partie des usines emploient les blooms, à la sortie du blooming, et les laminent en profils divers à des trains moyens à la chaude, ou le plus souvent après réchauffage.

Les usines dites de " transformation " qui reçoivent les blooms et les billettes des grandes aciéries pour la fabrication des petits fers et du fil ont cherché à obtenir des sections de plus en plus faibles afin de diminuer chez elles les frais d'installation, et, surtout, les frais de laminage par la suppression des trains ébaucheurs supplémentaires ( $\mathrm{B}$ ox $»)$ prenant des blooms de 4 à $6 \mathrm{~cm}$. de section.

Les grandes aciéries ont alors adopté la billette de $4 \mathrm{~cm}$. comme type commercial courant pour l'alimentation des petits trains et trains-machine. Cette dimension correspond en général à la dernière cannelure du blooming, elle représente la section minimum que l'on peut obtenir, sur une seule cage, en partant d'un gros lingot de 2.500 à $2.700 \mathrm{~kg}$.

Cette billette est encore très répandue et employée dans les usines fabriquant du fil, elle est reprise après réchauffage dans des fours continus, ébauchée dans un petit train Morgan de 4 à 6 cages, puis de là conduite au train finisseur où elle est serpentée aux différentes cages. Par suite de son adoption presque générale par les grandes aciéries, le prix de vente en était devenu assez avanta. geux; mais en ces dernières années, les usines qui consommaient spécialement ce demi-produit se sont bien vite rendu compte que le travail d'ébauchage aux petits trains, de la billette de $100 \times 100$ pour la ramener aux dimensions plus faible jusquà $11 / 2 \times 11 / 2$ était encore trop dispendieux et compliquait toujours les petits trains d'un dégrossisseur supplémentaire.

On s'est demandé s'il ne serait pas possible de produire à la mème chaude, à la suite du blooming ordinaire, de la billette à faible section en partant de lin- 
gots de 2.500 à $3.000 \mathrm{~kg}$. Si la solution était possible techniquement, on avait le sentiment que le prix de revient des billettes ainsi obtenues serait certainement inférieur au prix de la billette de mêmes dimensions sortant des ébaucheurs supplémentaires ou petits trains "Box ».

Le problème a été résolu d'une manière très ingénieuse et pratique par l'application des trains continus “ Morgan » pour la préparation de la billette de faible section. Pour arriver au résultat final, on a eu à vaincre plusieurs difficultés entre autres le cisaillage automatique des longues barres en tronçons ne dépassant pas $10 \mathrm{~m}$.

La description sommaire de deux installations appartenant à la Société Carnégie montrera les dispositions généralement adoptées pour ce genre de laminage.

\section{Ancienne installation de Duquesne pour le laminage de la billette moyenne, de la billette à faible section et des largets (fig. 8).}

Cette installation se compose en réalité de 5 trains ayant leur moteur propre et disposés d'une façon un peu spéciale.

$1^{\circ}$ Blooming. - Ce train est alimenté par de gros lingots, qui sont transformés en blooms de 200 à $220 \mathrm{~mm}$. de section; la longueur de table est plutòt faible, le nombre de cannelures en est limité. Le laminage à ce blooming est très rapide; la succession des lingots s'y fait d'une façon régulière et intensive; à l'arrière du blooming se trouve une cisaille qui sectionne les blooms en trois tronçons destinés à des produits différents.

$2^{\circ}$ Le train II qui fait suite au blooming est en quelque sorte un ébaucheur monté en trio ayant des cylindres de 650 à $700 \mathrm{~mm}$. de diamètre environ; le fonctionnement de ce train a beaucoup d'analogie avee l'ébaucheur à rails d'Edgar Thomson, le trio de South Chicago, de John Laughling, ete. Il est bien agencé et fonctionne très bien; des rouleaux à mouvements indépendants aux tabliers, des guides spéciaux et des dispositifs très ingénieux pour le retournement contribuent à faire de ce train un outil remarquable pour la préparation des ébauches.

Par suite des dispositions spéciales de la construction de ses tabliers, on peut laminer à ce train deux blooms à la fois, suivre aussi le débit du blooming et alimenter d'une façon continue les trains finisseurs en ébauches destinées à faire de la billette de $100 \times 100$, de la billette de $38 \times 38$ et des largets de 175 à $200 \mathrm{~mm}$. Le nombre des passages à ce trio est restreint de 7 à 9 au maximum.

$3^{\circ}$ Les trains III et IV ont chacun 3 cages montées en duo de $6300 \mathrm{~mm}$. de diamètre environ; chaque paire de cylindres, porte un petit nombre de cannelures; les tables de ces deux trains sont fixes, mais on passe de la cage 2 à la cage 3 (avant) du train III par un culbuteur comme au train à rails de SouthChicago; on opère de même pour passer de la cage 1 à la cage 2 du train IV.

Par suite de la disposition des cages, on lamine eu bas aux cages 1 et $3 \mathrm{du}$ train III de même qu'aux cages 1 et 3 du train IV; on lamine en haut aux cages 2 des trains III et IV. Ce mode de laminage comporte par suite des tables à rouleaux à des niveaux différents. 
F. CORVÉE

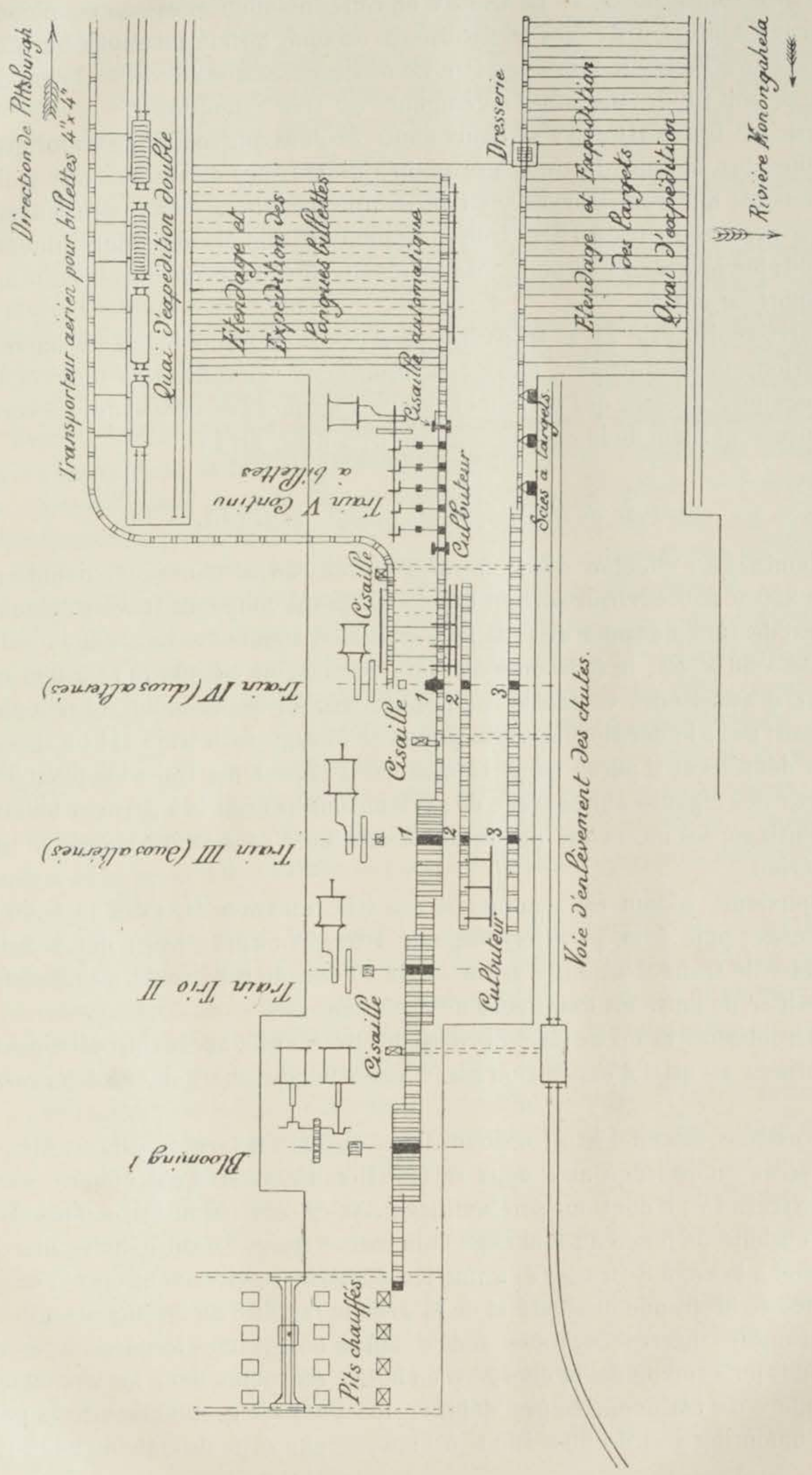

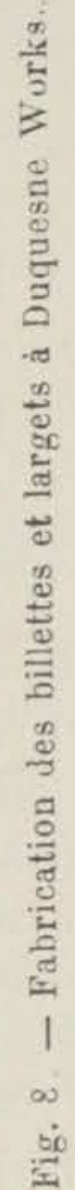


$4^{\circ}$ Train continu ${ }^{\circ} 5$. - - Le train $\mathrm{V}$ de cette installation est un train continu type Morgan à plusieurs cages transformant en cinq ou six passages les blooms du train III en billettes de faible section. Le fonctionnement de ce dernier laminoir bien que ce soit un des premiers de ce genre est très satisfaisant.

Entre les trains III et IV se trouve une cisaille qui sectionne en longueurs plus faibles les blooms destinés aux petites dimensions à laminer aux trains 3 et 4. A l'avant du train Morgan se trouve une cisaille à ébouter; enfin sur le côté et en arrière du train IV se trouve également une cisaille pour billettes de $100 \times 100$; les morceaux en sont enlevés et entrainés aux quais d'expédition par un transporteur aérien.

A l'arrière du train IV se trouvent quelques scies qui débitent les barres ou largets en longueurs multiples. Ceux-ci sont emmenés au refroidissoir ou conduits à une machine à dresser qui rectifie et polit les surfaces des îlots, qui ne sortent pas parfaitement plats de la cage finisseuse, $\mathrm{n}^{\circ} 3$ du train IV.

\section{Mode de laminage à ce groupe de trains.}

Le laminage s'effectue d'une façon générale de la manière suivante : un bloom de $220 \times 220$ environ sortant du blooming est coupé en trois tronçons; le premier est destiné à donner de la billette de $100 \times 100$, le deuxième de la billette de $38 \times 38$ à $50 \times 50$; le troisième donnera des largets de $171 \mathrm{~mm}$. à $200 \mathrm{~mm}$. de largeur à épaisseurs variables. Le premier bloom est ébauché au trio ébaucheur II sauf pour le dernier passage effectué à la cage 1 du train III où il reçoit sa section définitive; il passe sans laminage à la cage 1 du train IV puis il est entraîné par des ripeurs sur la ligne de rouleaux de la cisaille à grosses billettes. Après cisaillage, les morceaux ou fractions du bloom sont emmenés par le train porteur aérien.

Le deuxième bloom est également ébauché au train II, puis au train III (première cage) puis à la première cage du train IV ; après avoir été ébouté il s'engage dans le train Morgan pour en sortir à l'état de billette de faible section qui est cisaillée de suite en longueurs de 8 à $10 \mathrm{~m}$.

Un retroidissoir muni de ripeurs reçoit les barres qui, après refroidissement, sont entraînées au quai d'expédition placé vis-à-vis du chargement des grosses billettes.

Le troisième bloom destiné à donner des skelps ou largets est préparé au train II, reçoit un passage aux deux trains III et IV, puis il est culbuté sur le tablier R du train IV; il continue son laminage aux cages 2 (train IV) et (train III), puis il est culbuté de nouveau à la cage 3 du même train. Continuant sa marche il est terminé à la cage 3 (train IV) à une température relativement élevée ce qui est un indice de la grande intensité et de la grande rapidité du laminage en question. Les longues barres obtenues sont débitées en grandes longueurs par les scies à chaud, puis refroidies et chargées en bottes ou rames dans les wagons.

Ces différents trains-finisseurs débitent avec la plus grande facilité la production du blooming soit de 80 à $100 \mathrm{t}$. à l'heure; par suite de cette distribution 
de travail, un grand nombre de barres sont en prise avec les différentes cages correspondant à trois lingots dont le dernier est au blooming. Il n'y a aucun retard dans les différentes phases du laminage; la distribution du travail est du reste parfaitement organisée; le personnel est très nombreux et bien entraîné. On peut noter d'une façon spéciale le fonctionnement du train II qui est remarquable comme régularité et intensité.

L'enlèvement des chutes se fait à ces différents trains par des transporteurs latéraux; les rognures n'encombrent pas les cisailles.

Cette installation prise dans son ensemble représente pour l'époque à laquelle elle a été construite une application extrêmement intéressante du principe de laminage earactérisé par un blooming alimentant à la fois plusieurs industries de demi-produits; mais la section la plus intéressante de cette installation est sans conteste le train continu Morgan pour le laminage des petites billettes.

Train continu à billettes Morgan. - Ce train est actuellement bien connu de tous les ingénieurs de forge au moins dans ses grandes lignes de construction; il se répand de plus en plus dans les usines américaines non seulement pour la fabrication de la petite billette, mais aussi pour le laminage des petits plats, petits carrés, gros fil, et enfin, tend à se substituer aux petits trains actuels pour le laminage des petits ronds et autres profilés de faibles dimensions.

Le train V ou continu est un des plus anciens de ce genre; il est constitué par plusieurs cages duo successives à vitesses eirconférentielles croissantes où le laminage de la billette ogivale s'opère par retournement à $90^{\circ}$ à chaque passage par des guides héliçoïdaux à lunette placés à l'arrière de chaque cage.

On cherche à obtenir à chaque paire de cylindres des vitesses de sortie telles que la barre doit être légèrement tendue entre deux cages successives; on dresse pour chaque train un tableau des vitesses circonférentielles à chaque cage en fonction de la section des barres et par suite des allongements successifs que l'on se propose d'obtenir.

Dans quelques-uns de ces trains le diamètre des cylindres, va en décroissant de la cage finisseuse à la première cage, cela facilite l'augmentation progressive de la vitesse circonférentielle des cylindres, dont la vitesse angulaire croissante est donnée par les rapports des engrenages coniques de commande qui augmentent de la première cage à la cage finisseuse.

Les légères variations d'épaisseur provenant des différences de température des différentes barres ainsi que les écarts dans les vitesses circonférentielles provenant des variations sur le diamètre des cylindres soumis au tournage sont compensées aux différentes eages par un réglage convenable effectué au moyen de coins placés dans les cages. Ces coins agissent sur l'écartement des cylindres et par suite sur les allongements.

En ce qui concerne le laminage de la billette, des différences peu sensibles entre la vitesse de sortie d'une barre et celle d'entrée à la cannelure suivante, n’ont pas une grande importance ; il peut mème se présenter une légère compression faisant cintrer les barres principalement aux derniers passages où la section est faible. Mais pour le laminage des petits ronds, du fil, des feuillards, cornières 
et petits profilés la proportionnalité des vitesses circonférentielles aux allongements à chaque cage doit être respectée en tenant compte de la petite différence qui existe entre la vitesse propre aux barres quittant une paire de cylindres et la vitesse moyenne circonférentielle de ces derniers pour un profil donné.

Pour le train $\mathrm{V}$ en question, les allongements pour les billettes sont assez élevés; la vitesse de sortie à la dernière cannelure atteint $2^{\mathrm{m}}, 2 \mathrm{~g}$ à $2^{\mathrm{m}}, 50$ à la seconde avec des cylindres ayant $350 \mathrm{~mm}$. environ de diamètre.

A la suite d'un premier examen, cette installation paraît compliquée; pour la fabrication d'un demi-produit comme la billette, la force motrice ne parait pas devoir être inférieure à 1.000 et peut-être 1.200 chvx, soit de 200 chvx environ par élément du train, ce qui représente une somme de résistances à vaincre assez élevée à chaque passage. En revanche, ce laminoir représente bien le train continu par excellence ne nécessitant pour son fonctionnement aucun ouvrier de forge en dehors du chef lamineur; de plus la production par $24 \mathrm{~h}$. d'un tel appareil est considérable et a pu atteindre $1.000 \mathrm{t}$. en petites billettes.

L'installation d'ensemble du train Morgan comprend quatres lignes de longrines parallèles; la première et la deuxième supportent les paliers d'angle pour l'arbre principal transmettant par des engrenages coniques à rapports différents, le mouvement à des arbres transversaux; la troisième longrine supporte les petites cages à pignons fermées type Morgan; la quatrième ou longrine principale porte les eages à cylindres à table très courte portant quelques cannelures; le diamètre des cylindres ne dépasse pas en général $350 \mathrm{~mm}$. L'attaque se fait par les cylindres inférieurs au moyen de pignons, allonges, manchons comme à l'ordinaire.

La machine motrice attaque l'arbre principal à la vitesse moyenne de 75 à 8. tours à la minute; les variations de régime produites par l'engagement successif des barres aux différentes cages sont corrigées par un volant à jante très lourde placésur l'arbre de la machine. La vitesse relativement faible aux premières cages correspondant aux plus fortes sections et où les allongements sont élevés, explique le peu de ruptures constalées à ces trains continus; du reste les pignons coniques transmettant le mouvement aux dilférentes cages sont largement calculés et constitués d'un acier spécial de première qualité. Le fait d'engager les barres à chaque cage à une vitesse sensiblement égale à la vitesse d'entrainement des cylindres suivants, explique également la faiblesse des chocs constatés au moment du contact avec les cylindres.

Ce système de laminage constitue un progrès immense sur le train Bedson ou ses dérivés et réalise ce que l'on a fait de mieux dans la voie du travail continu en ces dernières années; il fait le plus grand honneur à la maison Morgan qui en a eu l'initiative et n'a cessé de le perfectionner.

A l'origine de ces installations, une grande difficulté s'est présentée au sujet du sectionnement des très longues barres produites par ce train; on ne pouvait songer à les entrainer aux scies ou cisailles par de longues lignes de rouleaux à la façon ordinaire. On créa alors la cisaille verticale oscillante automatique effec. tuant le cisaillage à chaud derrière la cage finisseuse sans arrèter la translation 
ou mouvement d'avancement des barres cheminant à la vitesse moyenne.de $2^{\mathrm{m}}, 50$ à la seconde. Ce remarquable ontil dù à M. Edward, ingénieur de la maison Morgan, est constitué par un bàti vertical oscillant autour de sa base où il est articulé, il porte deux couteaux, l'un fixe, l'autre supéricur mobile.

Pendant que s'elfectue le déplacement de la longueur de billette à couper, la cisaille est au repos et inclinée du côté de la cage linisseuse. Au moment où l'extrémité de la billette vient toucher le butoir de la cisaille, un dispositif attenant au butoir ouvre l'admission de vapeur dans un cylindre placé sous le sol dont le piston est relié par des bielles au bàti de la cisaille à sa partie supérieure; celui-ci est ramené brusquement en arrière. En même temps le porte-lame supérieur ou couteau qui est guidé à la partie supérieure du bâti et est relié par des bielles à un point fixe de la plate-forme descend et effectue le cisaillage. La billette cisaillée tombe sur les rouleaux, abandonne le butoir et n'agit plus sur le dispositif relié au distributeur; celui-ci reprend sa position primitive, oblige par cela même le bâti de la cisaille à revenir en avant au moyen du piston et de ses bielles de rappel ; pendant cette manœuvre presque instantanée la billette trouvant devant elle le couteau supérieur brusquement relevé, continue d'avancer dans la lunette formée par l'échancrure des lames. Le cisaillage s'opère ainsi « au vol ") suivant l'expression américaine, la coupe est franche sans pliage ni torsion de l'extrémité, les longueurs sont à très peu près semblables comme on en peut juger par la vue des billettes reposant sur le refroidissoir.

\section{Laminoir continu à billettes annexé à un blooming.}

(Nouvelles installations de Duquesne (fig. 9) (1).

La Société Carnegie se basant sur l'expérience acquise avec l'ancienne installation a fait une nouvelle installation motivée par la création de ses laminoirs pour fers marchands.

L'installation comprend deux trains :

$1^{\circ}$ Un blooming de $1.000 \mathrm{~mm}$. de diamètre environ;

$2^{\circ}$ Un train continu Morgan à billettes de $350 \mathrm{~mm}$.

Les lingots venant du Martin sont enfournés dans les pits placés à l'extrémité de la halle du blooming; ils en sont retirés comme à l'ordinaire par un pont roulant avec tenailles rigides. Ces pits sont très bien installés et constituent un type que l'on retrouve dans les nouvelles usines avec plusieurs lingots par cellule, quatre cellules par four et plusieurs fours réunis en batteries qui sont alimentés par le gaz naturel dans la région de Pittsburgh et au gaz de gazogène dans les usines situées dans la région des grands lacs. La dépense de combustible ramenée à la consommation de houille varie de $75^{\prime \prime}$ à $90^{\prime \prime}$ de houille par tonne et lingots enfournés; le déchet de fer varie de 0,60 à $0,80 / 0$.

Le train blooming est un des plus récents des usines de la région de Pitts.

(1) Une description très détaillée de cette installation a été donuée par l'Iron Age dans ses numéros des 1 or et 8 jauvier 1903 . 
burgh; il est caractérisé par la grande robustesse de ses organes; sa table est relativement faible et comporte un petit nombre de cannelures; la levée du cylindre supérieur est considérable, on passe plusieurs fois dans les premières cannelures. L’équilibrage du cylindre supérieur est réalisé par des pistons hydrauliques fixés à la partie supérieure des cages; on constate de plus en plus dans les nouveaux trains la disparition du système d'équilibrage par pistons hydrauliques placés dans la fosse des trains. Le mouvement des vis se fait par une longue crémaillère actionnée par piston hydraulique placé sur la cage à pignons. Les longrines pour tables à rouleaux de même que les rouleaux d'entraînement sont très robustes; toutes les dimensions en sont largement calculées.

La machine réversible de $1.400 \times 1.525$ n'attaque pas directement le train; des engrenages réduisent la vitesse moyenne du blooming à 30 ou 40 tours; on constate dans le dégrossissage des lingots aux première cannelures des réductions d'épaisseur très élevées principalement aux premiers passages.

Les blooms ou rames laminés à ce blooming sont éboutés et confiés à une cisaille très puissante coupant facilement 12 décimètres carrés ; elle comprend des dispositifs de détails bien compris qui permettent une grande rapidité dans les opérations du cisaillage. Les chutes sont enlevées par des vagons spéciaux venant se placer près de la cisaille.

Ce blooming alimente à la fois le train continu pour petites billettes et fabrique également des blooms et brames qui sont expédiés de suite ou mis en stock dans un magasin situé sur le côté de la halle du train continu. La production peut être estimée de 1.600 à $1.800 \mathrm{t}$. pour 24 heures; il y a lieu de noter ici que la plus grande partie de cette production est constituée par des billettes méplates de $150 \mathrm{~mm}$. $\times 120 \mathrm{~mm}$. environ destinées à l'alimentation du train continu.

Train continu d̀ billettes de $350 \mathrm{~mm}$. - Ce laminoir comprend de neuf à dix cages disposées à la suite l'une de l'autre comme dans l'installation précédemment décrite. La machine motrice compound avec un cylindre horizontal et un cylindre vertical développe de 1.700 à 1.800 chvx. et tourne avec une vitesse de 85 à 95 tours. Cette disposition de machine motrice se rencontre actuellement dans quelques usines et a été adoptée dans les souffleries de Lakawanna.

La première paire de eylindres peut prendre la billette sortant du blooming qui est laminée succeessivement aux cages du train continu et transformée en petites billettes de $38 \times 38$ à $50 \times 50$ de la même chaude que le lingot lui-même. Les allongements sont très élevés comme on peut s'en rendre compte par le calcul; la billette entre dans la première cage avec une vitesse moyenne de $0^{\mathrm{m}}, 35$ à $0^{\mathrm{m}}, 40$ et sort de la dernière eage avec une vitesse approximative de $2^{\mathrm{m}}, 50$ à $2^{\mathrm{m}}, 65$ à la seconde correspondant à 150 tours environ à la cage finisseuse. Le retournement des barres s'effectue comme cela a été indiqué précédemment à la sortie des cannelures par de forts guides tubulaires hélicoïdaux fixés très solidement aux sommiers ou traverses des eages.

L'écartement entre les différentes paires de cylindres quoique aussi réduit que possible n'est pas constant, il va en augmentant des premières cages aux 
F. CORVÉE

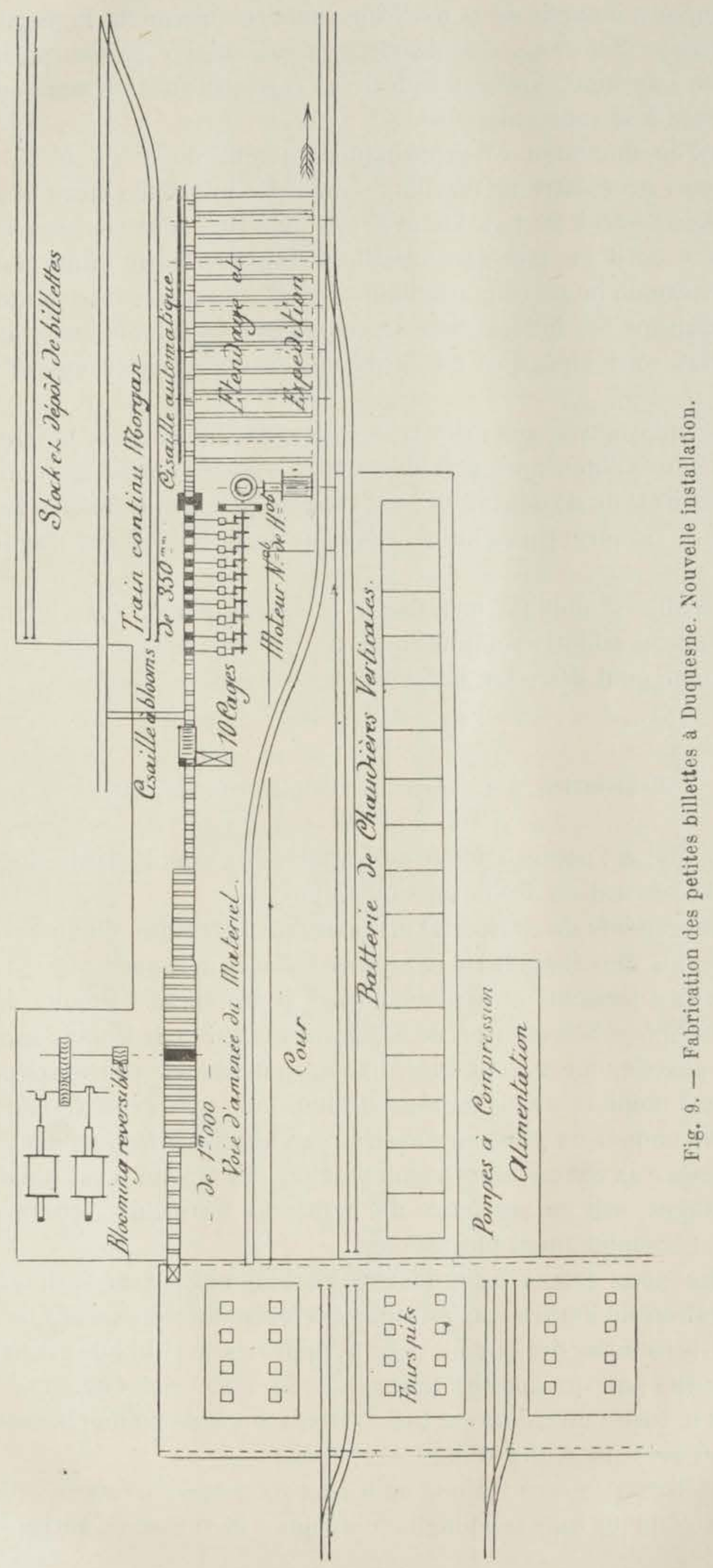


dernières comptées à partir de la machine motrice voisine de la cage finisseuse où se fait l'attaque du train. Cet écartement est motivé par la position et les dimensions des engrenages coniques dont les rapports vont en augmentant de la cage ébaucheuse à la cage finisseuse.

Le temps de laminage est approximativement de $1^{\prime} 40^{\prime \prime}$ à $2^{\prime}$, en tenant compte du temps nécessaire au cisaillage, du léger intervalle entre deux blooms. 0 n peut laminer de 50 à $60 \mathrm{t}$. à l'heure. Ces billettes sont coupées en grandes longueurs de 8 à $10 \mathrm{~m}$. par une cisaille Edwards faisant une coupe toutes les 4". A l'arrière de la cisaille oscillante automatique se trouve une ligne de rouleaux conduisant les billettes sur des chantiers de refroidissement et d'étendage; puis elles sont ripées et chargées sur wagons placés en contrebas des chantiers.

On comprend qu'avec une installation aussi grandiose pour la fabrication de la billette, on pense obtenir un prix de revient excessivement bas dont les frais propres de fabrication ne dépassent pas 1 dollar par tonne depuis l'arrivée du lingot venant de l'aciérie jusqu'au chargement de la billette sur wagon au quai d'expédition.

Le prix d'une semblable installation est très élevé, mais étant donnée l'énorme production annuelle obtenue avec un tel train, en aucun cas l'amortissement par tonne ne peut dépasser $0^{\text {dol }}, 25$.

\section{Fabrication des largets à Vandergrift (fig. 10).}

On a appliqué à l'usine à tôles de Vandergrift une variante du laminage continu pour la fabrication des largets ou bidons.

L’installation générale comprend en réalité trois trains : un blooming réversible et deux trains duo ébaucheur et finisseur placés à la suite.

Le blooming possède cinq cannelures, il prend des lingots de 2.200 à $2.300 \mathrm{~kg}$. ayant $500 \times 500$ environ de section à la base; ces lingots sont chauffés dans des pits chauffés au gaz naturel, ils sont amenés en plusieurs passages à l'état de blooms méplats ayant comme dimensions approximatives $185 \times 125$; ces blooms sont coupés en plusieurs parties par une cisaille puis sont successivement relaminés à la chaude aux trains précités. Le travail n'est ainsi distribué qu'en six passages, soit un par paire de cylindres finisseurs, on transforme le bloom en larget complètement terminé.

Chacun des deux trains faisant suite au blooming, l'un était en quelque sorte le préparateur de l'autre. Le finisseur est formé de trois paires de cylindres placées à la suite les unes des autres et très rapprochées. Chaque groupe de trois cages est actionné par un moteur horizontal de 1.200 à 2.500 chvx. L'écartement des deux groupes de trains est ealculé de façon à ce qu'une barre ne puisse être en prise quavec un seul groupe.

Dans l'installation de ces trains, on s'est préoccupé d'avoir des vitesses circonférentielles à chaque cage sensiblement égales à la vitesse de sortie des barres 


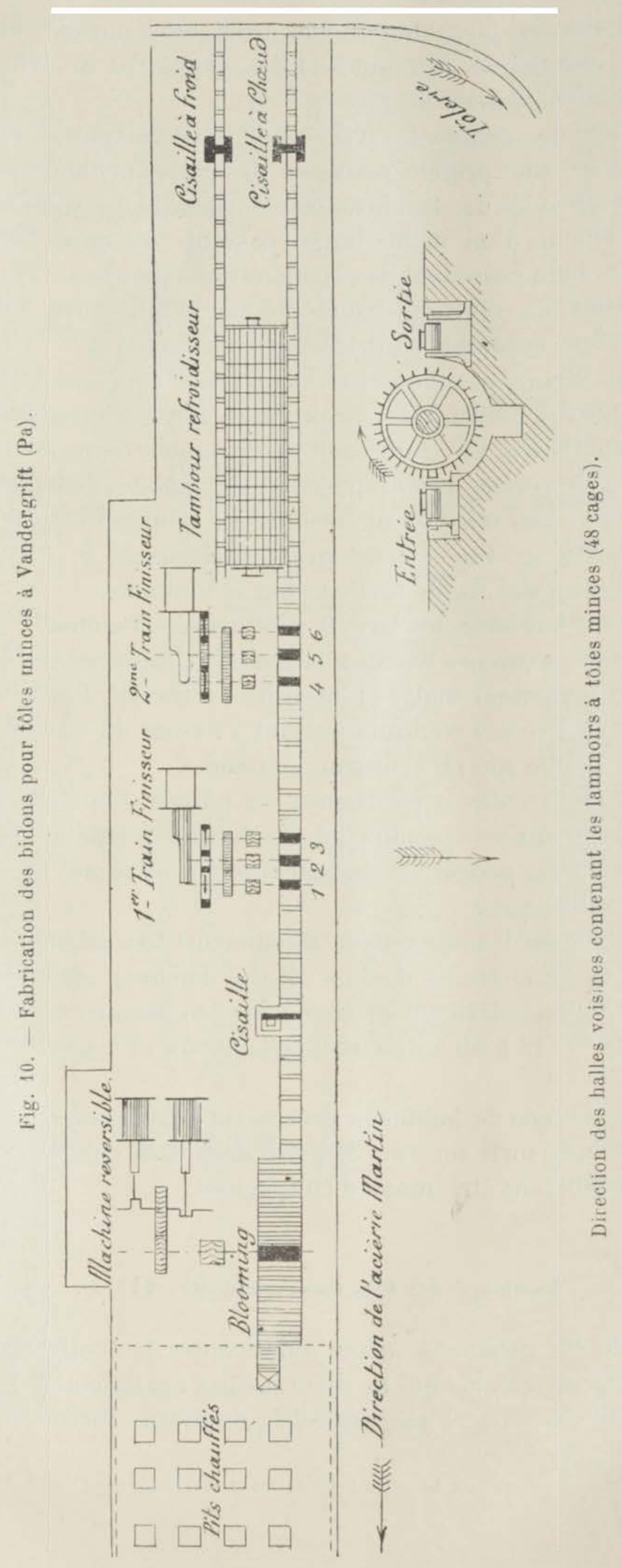


de la cage précédente; on a réalisé ainsi avec trois cages un système de train continu dont les vitesses circonférentielles croissantes ont été obtenues à la fois par des vitesses angulaires différentes et des diamètres de cylindres croissant avec le degré d'amincissement des barres.

Le mouvement de serrage des vis se fait avec volants et plateaux divisés; on peut obtenir avec une grande précision des plats ou bidons aux dimensions demandées par le service des trains à tôles minces; la question se complique ici du fait que les blooms d'un même lingot passent l'un après l'autre aux trains finisseurs, et par suite possèdent des températures inégales à la cannelure finis. seuse. La précision du réglage permet d'éviter cette cause d'irrégularité, les bidons pris dans leur ensemble sont très bien laminés.

A la suite du dernier groupe se trouve un grand tambour refroidisseur d'une construction originale pouvant contenir vingt-cinq à trente largets sur sa demi circonférence supérieure. Ce tambour d'une longueur approximative de $25 \mathrm{~m}$. est constitué par des cercles assez rapprochés portant des dents très bien alignées et espacées de 150 à $200 \mathrm{~mm}$.; le mouvement du tambour est calculé de manière à recevoir les barres au fur et à mesure du laminage; le refroidissement est activé par une pluie d'eau froide tombant sur le tambour.

A l'arrière du tambour, les largets sont libérés et emmenés par groupe à la cisaille où on coupe plusieurs barres à la fois ; le cisaillage est fait très soigneusement et très exactement malgré l'intensité du travail. Dans le cas de bidons épais, la ligne primitive des rouleaux passant à l'avant du tambour est prolongée et aboutit à une cisaille spéciale coupant à chaud.

Les bidons sont enlevés par rames au moyen d'un pont roulant qui les dépose sur des wagonnets spéciaux les conduisant à l'atelier de laminage; les tenailles spéciales pour prendre et manutentionner les rames de bidons sont bien comprises et très pratiques.

Le fonctionnement de toute cette installation est très satisfaisant; on a laminé dans ce groupe de trois trains de 25 à $28 \mathrm{t}$. à l'heure; mais on produira bien davantage avec l'augmentation projetée de l'aciérie Martin; cette installation doit pouvoir produire de 35 à $40 \mathrm{t}$. de largets ou bidons à l'heure avec la plus grande lacilité.

Il y a dans ce mode de laminage précité un acheminement au train continu qui serait en quelque sorte un train Morgan de grandes dimensions à six cages, la dernière eage pouvant être montée en spataces.

Laminage des fers marchands (fig. 11) (1).

On voit dans les anciennes usines américaines de nombreux trains pour le laminage des fers marchands qui ne diffèrent pas sensiblement comme ensemble des trains européens; mais des dispositifs de détails facilitent à un personnel

(1) Une dezcription très complète de cette installation a été donnée parl'Iron Age dans ses numéros des 1 er et 8 janvier 1903 . 


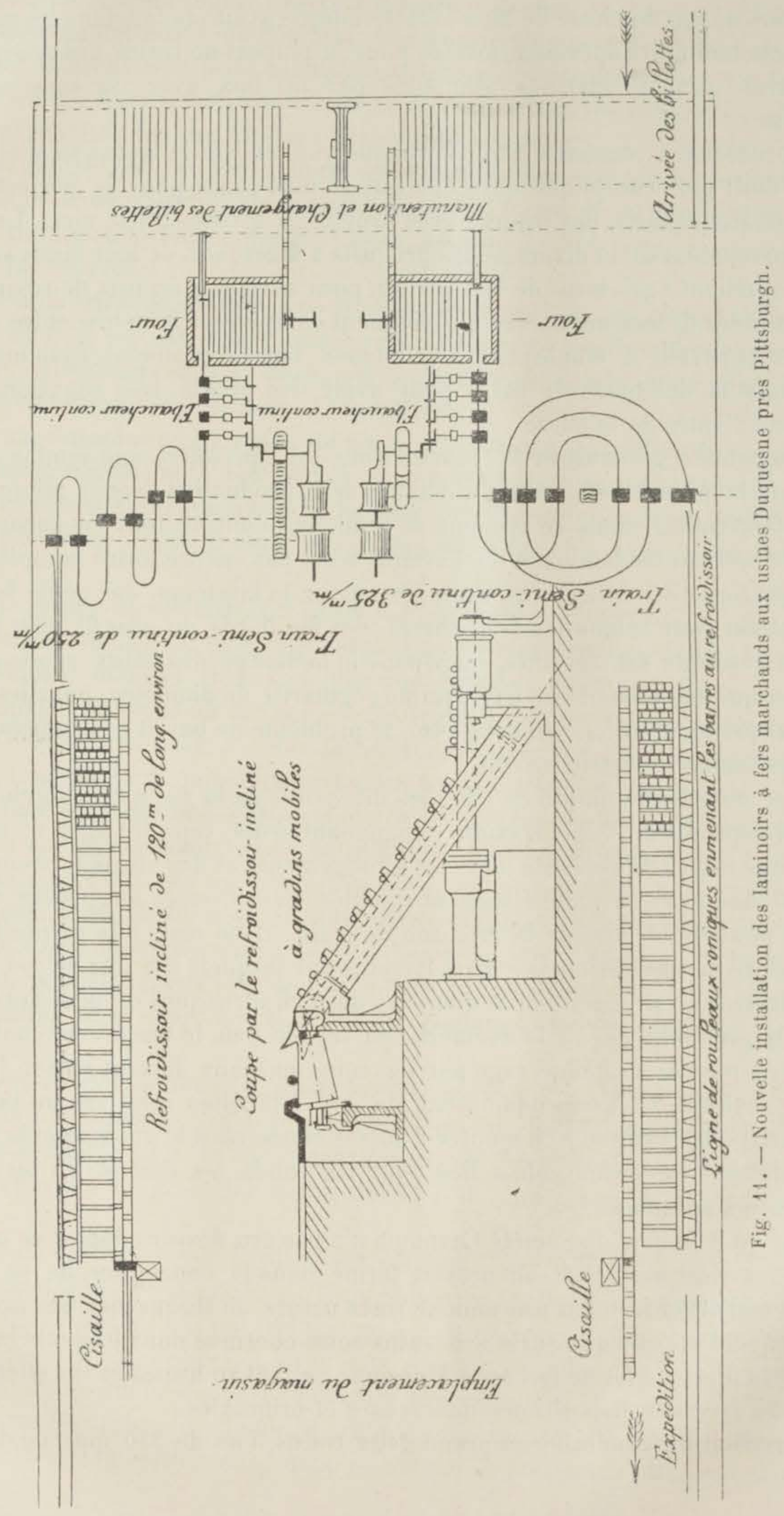


ouvrier de premier ordre l'obtention de productions élevées. Jusqu'à ces dernières années, peu de chose de bien caracléristique avait été fait dans les installations de ces laminoirs spéciaux, formés pour la plupart de trains finisseurs montés en trios ou duos alternés alimentés par un box avec ou sans moteurs spéciaux.

Le problème à résoudre pour faire quelque chose de nouveau n'était pas facile; il était plus compliqué que ceux qui se sont posés pour le perfectionnement des trains à rails, des trains à gros profilés, à grosses tôles, etc... Les difficultés provenaient de la diversité des produits à fabriquer, de leur faible section; elles provenaient également de l'obligation pour arriver à un prix de revient très bas, de laminer de longues barres qui viennent compliquer la nature et les dimensions des appareils à employer; ces longues barres motivent également des dispositions et des tours de main pour avoir des profils très réguliers d'une extrémité à l'autre de la barre.

On a pensé à généraliser le train continu qui à première vue sembla donner la solution la plus satisfaisante du maximum de production avec le minimum de frais de fabrication, mais ce système de laminage continu tout en apparaissant comme le système de laminage de l'avenir, n'est pas encore entré complètement dans la pratique des forges aux États-Unis pour le laminage des petits fers. On a bien obtenu pour le laminage des carrés, des feuillards, du gros fil, pour le tréfilage des résultats satisfaisants, mais pour le laminage des petits ronds pour le commerce qui demandent une très grande régularité de diamètre, pour les petites cornières, pelits fers à $\omega$, vitrages, etc., le problème ne paraît pas complètement résolu, au moins industriellement.

On se rend bien compte en effet des dificultés de la solution cherchée pour obtenir des profils de faible section s'engageant seuls, sans traction ni refoulement, d'une cage à la suivante avec des allongements et des vitesses circonférentielles à chaque cage judicieusement calculés. Les vitesses angulaires établies une fois pour toutes lors de la création du train, il ne reste à la disposition du personnel de la fabrication que deux facteurs sur lesquels il a une action plus ou moins directe; en premier lieu le diamètre des cylindres que l'on peut faire croissant de la première cage à la dernière; en second lieu le tracé convenable des cannelures réglées à chaque cage par les coins spéciaux faisant varier l'écartement des cylindres. Indépendamment de ces difficultés relatives au laminage proprement dit, viennent s'en ajouter d'autres concernant le guidage et le retournement des barres comme cela a lieu pour les ronds, les carrés, les fers à $\perp$ et quelques fers spéciaux.

Quoi qu il en soit, la Société Carnegie n’a pas cru devoir adopter ce mode de laminage et s'est arrêtée à un moyen terme dans la conception de sa remar. quable installation formant une annexe à ses usines de Duquesne. Ses nouveaux laminoirs sont en quelque sorte des trains semi-continus dans lesquels la préparation dela fusée de fer se fait au "Morgan »; quant au finissage il s'effectue par serpentage avec des dispositions ingénieuses et originales.

L’installation générale comprend deux trains, l’un de $330 \mathrm{~mm}$. de diamètre 
pour les cylindres finisseurs; l'autre plus faible destiné au laminage des petits fers possède des cylindres de $2.50 \mathrm{~mm}$ Ces deux trains sont renfermés dans une vaste halle dont l'extrémité est occupée par les chantiers de billettes, les fours et les deux trains; les refroidissoirs spéciaux en occupent les côtés; le centre est réservé au magasinage des longues barres non cisaillées.

Train de $330 \mathrm{~mm}$. - Ce laminoir est actionné par une machine tandem de 1.200 à 1.500 chvx. faisant approximativement 85 tours; il est contitué par deux trains, l'un servant d'ébaucheur, l'autre servant de finisseur, ce dernier est actionné par courroie.

Le petit ébaucheur continu type Morgan comprend quatre cages, il prend de la billette de 7.5 à $100 \mathrm{~mm}$. et la transforme en ébauche convenable pour le profil à laminer; cet ébaucheur n’a rien de particulier, il est semblable à ceux décrits précédemment pour la fabrication de la billette.

Le train finisseur se compose de six cages formant en réalité deux groupes de trois cages reliés entre eux par la eage à pignons; ces deux groupes sont placés à des niveaux différents afin de faciliter aux barres leur serpentage spécial. Les trois premières cages travaillent en duo inférieur, les trois autres en duo supérieur.

Le serpentage de ces barres à forte section étant très difficile à effectuer à la main on s'est inspiré du mode de serpentage des carrés aux ovales dans les trains-machines ; mais au lieu d'adopter des cheneaux demi-circulaires on s'est servi du dallage à l'avant et à l'arrière du train finisseur, lequel présente des parties en creux terminées par des courbes destinées à guider la barre. On a adopté alors un mode de laminage concentrique, le diamètre des boucles et par suite des demi-circonférences directrices dans les plaques de dallage allant en augmentant au fur et à mesure que décroît la section de la barre.

Le laminage s'effectue en général de la façon suivante: la barre ébauchée sortant du petit train con tinu est conduite par un chenal à l'arrière du train finisseur où par l'effet de la première courbe directrice elle s'achemine à la cage 3 ( $1^{\text {er }}$ groupe), puis aux cages $4,2,5,1$ et enfin à la finisseuse (cage 6 ) où elle sort dans un chenal pour aller sur la ligne de rouleaux du refroidisseur spécial incliné.

Le laminage à ces différentes cages est très rapide, il convient très bien pour la nature des fers laminés à ce train en ayant en vue une forte production; les carrés et les ronds de 15 à $3 . ّ \mathrm{~mm}$. sont obtenus en barres de près de $100 \mathrm{~m}$. de longueur en partant de grosses billettes de 50 à $75 \mathrm{~mm}$. ayant de 8 à $10 \mathrm{~m}$. de longueur.

Ce train de mème que son voisin est alimenté par des fours spéciaux du système Morgan, qui a reçu aux États-Unis plusieurs applications. Ces fours peuvent ètre chauffés soit au gaz naturel soit au gaz de gazogène ; ils constituent en quelque sorte des fours continus à large sole pouvant chauffer des billettes ayant jusqu'à $10 \mathrm{~m}$. de longueur à une température très régulière. De même que dans les autres fours continus, le cheminement des billettes se fait sur des chenets refroidis, l'entrée et la sortie s'effectuent au moyen de pousseurs; ils sont placés 
très près des ébaucheurs afin de diminuer le temps de refroidissement pour la partie de la billette qui se laminera en dernier lieu et qui est encore dans le four lorsque la première extrémilé est déjà en prise avec les premiers cylindres du train continu. Ces fours sont en tous points remarquables comme construction et fonctionnement, leur capacité de production est considérable, elle peut atteindre de 4 à $500 \mathrm{t}$. par vingt-quatre heures; un four peut prendre de 75 à 8.5 billettes de $50 \mathrm{~mm}$. et un nombre plus considérable de billettes plus faibles; la vitesse de défournement pouvant atteindre de 80 à 100 billettes à l'heure, chaque billette séjourne dans le four de quarante-cinq à soixante minutes environ.

La consommation de gaz naturel ou de gaz de gazogène ramenée à la quantité de houille correspondante ne dépasse pas $100 \mathrm{~kg}$. par $\mathrm{t}$. de produits finis dans les conditions les plus défavorables. Un immense chantier desservi par des ponts roulants sert a décharger, étaler et distribuer aux fours la billette venant des trains continus précédemment déerits.

La vitesse de laminage à la sortie de la cage finisseuse paraît être de $2^{\mathrm{m}}, 50$ à $2^{\mathrm{m}}, 7.3$ à la seconde ; la production de ce train dans les petits échantillons est de 200 à 240 t. et de 360 à $400 \mathrm{t}$. avec les gros échantillons.

Avec de telles productions journalières et d'aussi longues barres à manutentionner, la question des opérations faisant suite au laminage a dù être solutionnée d'une manière toute différente de celle pratiquée jusqu'alors dans les anciennes installations.

En admettant des barres ayant $100 \mathrm{~m}$. de longueur et 100 billettes à l'heure (vitesse maximum de défournement) cela représente $10.000 \mathrm{~m}$. de fers à étendre, dresser, refroidir, cisailler et botteler en une heure. Jusqu'alors on s'était contenté d'effectuer à la main ces différentes manutentions sur des barres dont la longueur n'excédait pas $40 \mathrm{~m}$.; on a dù chercher autre chose pour le laminoir en question.

Dans l'installation de Carnegie, on a eréé de toutes pièces un outillage tout à fait remarquable comme conception, qui est caractérisé par un refroidissoir incliné et à grandeurs mobiles.

Ce refroidissoir se compose en principe de deux lignes de rouleaux établies à des niveaux différents, la ligne supérieure sert à conduire les barres du train au refroidissoir, l'autre ligne ou ligne inférieure conduit les barres froides à la cisaille; ces deux lignes de rouleaux sont réunies par le refroidissoir incliné proprement dit. Cet appareil est constitué par des traverses s'appuyant sur les longrines intérieures des deux lignes de rouleaux. Entre ces traverses se trouvent des barres mobiles formant de véritables rateliers, à dents alternées espacées de quelques centimètres formant ainsi des supports aux barres en cours de refroidissement; ces rateliers sont manœuvrés par un càble relié à chacun d'eux et qui leur fait subir dans un sens ou dans l'autre un angle de rotation qui produit l'éclipse ou met en saillie au-dessous du niveau des traverses les divers supports attenant aux rateliers.

La ligne de rouleaux supérieure présente quelques particularités assez ingénieuses; les rouleaux de la ligne supérieure ont une forme conique; ils sont 
montés de manière à ce que leur génératrice supérieure soit horizontale et émerge des plaques de recouvrement, la grande base est tournée et orientée à l'opposé du refroidissoir. De plus l'extrémité de ces rouleaux peut se déplacer sur la longrine extérieure, de manière que les axes de tous les rouleaux puissent prendre des inclinaisons positives ou négatives par rapport à des plans perpendiculaire aux Jongrines passant par l'extrémité fixe. En donnant par un câble, reliant tous les paliers mobiles, une obliquité à tous ces rouleaux en sens inverse du mouvement de translation du train au refroidissoir, on oblige la barre à cheminer en s'appuyant progressivement sur la grande base des rouleaux avec une vitesse de translation de plus en plus grande au fur et à mesure qu'elle se rapproche de cette arête.

Le rebord des plaques de recouvrement empêche la barre de sortir des rouleaux coniques et forme comme un angle de dressage pour les barres encore chaudes.

L'inelinaison brusque des rouleaux dans le sens contraire et le renversement de leur mouvement de rotation porte rapidement la barre sur le petit côté du còne où de petits leviers culbuteurs la font glisser sur la première rangée des rateliers. A chaque mouvement de rotation des rateliers, les barres glissent sur le plan incliné d'un intervalle de dents, d'une manière très régulière tout en restant parallèles entre elles, et arrivent ainsi au bas du plan incliné par le seul effet de la pesanteur, suffisamment froides pour ne pas se déformer ; elles se rassemblent sur la table précédant les rouleaux inférieurs où elles sont poussées par des pousseurs et de là conduites à la cisaılle.

En marche normale, le refroidissoir est complètement chargé de barres en refroidissement; le fonctionnement de cet appareil est continu et suffit largement au débit du train. Cet appareil en apparence très compliqué, de construction assez coùteuse, résoud parfaitement le problème du dressage et du refroidissement des fers marchands, laminés en longues barres et fabriqués avec une marehe intensive.

A la suite du refroidissoir se trouve une cisaille débitant les fers aux longueurs demandées par le commerce, on en forme des paquets ou bottes liées très solidement puis chargées sur vagon par un pont-roulant.

Train de $250 \mathrm{~mm}$. - Ce train voisin et symétrique du précédent est plus petit, il est agencé spécialement pour le laminage des petits fers. Il se compose d'un petit ébaucheur continu type Morgan prenant des billettes de $38 \times 38$ à $50 \times 50$ de section; puis d'un train finisseur à six cages agencé d'une façon particulière et un peu nouvelle.

Les six cages forment en réalité trois petits trains parallèles ayant des vitesses différentes et en retraite les uns par rapport aux autres ; de plus chaque cage d'une même ligne possède une vitese circontérentielle différente par suite des différences de diamètres du cylindre. De cette façon la vitesse de sortie des barres croît de la $1^{\text {re }}$ à la $6^{\text {e }}$ cage ; toutes les cages de ces trois petits trains sont montées en duo.

Une des particularités intéressantes de ce train, e’est la commande des trois 
trains finisseurs par une seule courroie passant successivement sur les poulies réceptrices et les enveloppant convenablement par une disposition spéciale avec le secours de rouleaux tendeurs.

Étant donnée la faible distance des axes de ces trois trains, il eût été difficile d'imaginer une solution répondant mieux à cette disposition un peu spéciale; on évite ainsi les relais de poulies à eâbles habituellement employés dans les tràins à fil.

Les longues billettes sont réchauffées dans un four analogue à celui précédemment décrit pour le train de $330 \mathrm{~mm}$., puis dégrossies sous la forme de petites ogives de 20 à $23 \mathrm{~mm}$. environ. Ces ébauches sont serpentées ensuite aux trains parallèles où elles sont transformées en petits fers; le laminage est très rapide, les barres restent à une température convenable et très régulière. A la sortie de la $6^{\text {e }}$ cage les fers sont conduits par un chenal à un refroidissoir incliné du mème genre que celui du train de $330 \mathrm{~mm}$.

La production de ce petit train est également variable avec la nature et les dimensions des échantillons laminés; on peut l'estimer de 150 à 200 t. par 24 heures.

En se référant aux données qui précèdent, et en prenant pour base le prix du lingot Martin estimé dans les devis précédents à $14^{\mathrm{dol}}, 500$, le prix de revient des demi-produits peut s'śtablir comme suit :

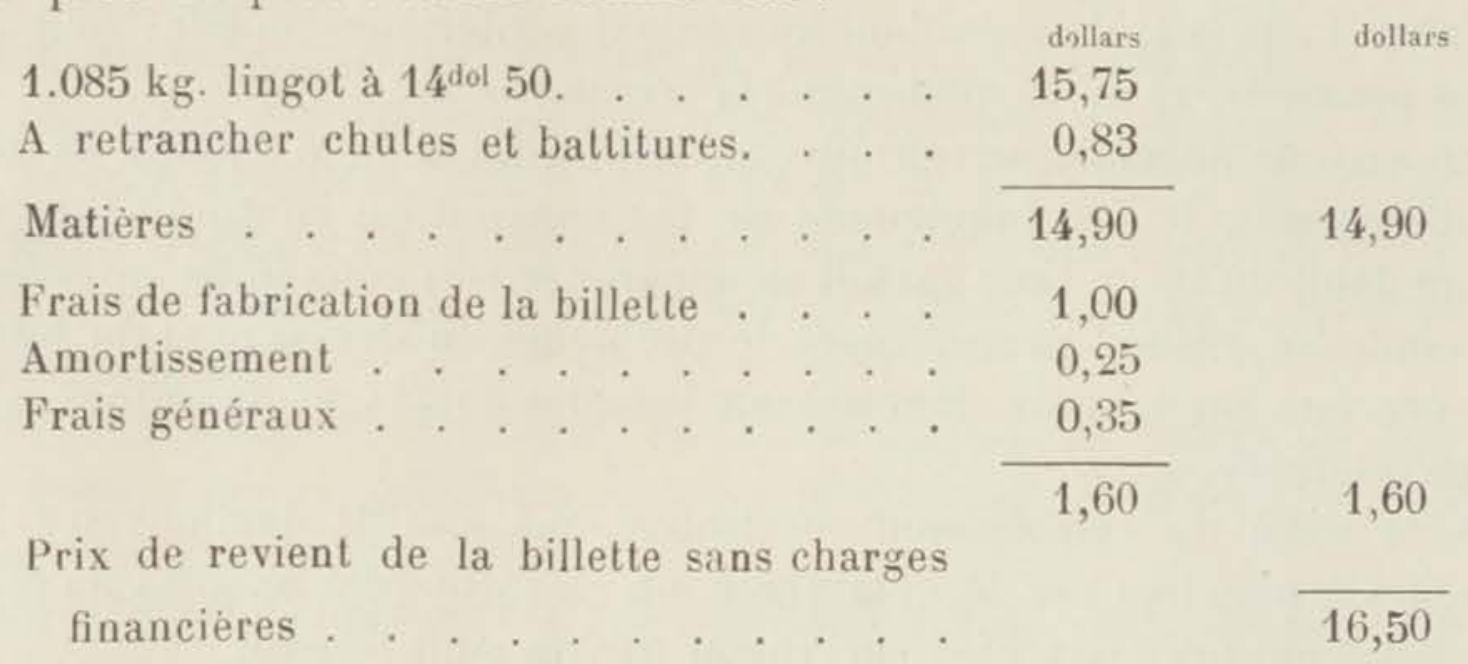

Cette billette était vendue de $17^{\mathrm{dol}}, 50$ à $21^{\mathrm{dol}}, 50$ en octobre 1904 .

Le prix de revient de la billette de $100 \times 100$ établi sur les mêmes bases, serait de 150 , 75 à 96 dollars.

Le prix du skelp ou largé peut ètre estimé dans les mèmes conditions de 16 dollars à $16^{\text {dol }}, 50$. 